\title{
Flux-to-Voltage Transfer Function of the Series-SQUID Array With Grain-Boundary Josephson Junctions
}

\author{
J. T. Jeng, H. C. Hung, C. R. Lin, C. H. Wu, K. L. Chen, J.-C. Chen, H. C. Yang, S. H. Liao, and H. E. Horng
}

\begin{abstract}
The factors affecting the flux-to-voltage transfer function of the series-SQUID array with grain-boundary Josephson junctions were investigated. By using the proposed criticalcurrent-modulation model, it is found that the voltage swing of the series-SQUID array is suppressed greatly by the spread in critical currents of Josephson junctions. For the inductance $L=80 \mathrm{pH}$ and the logarithmic average critical current $2 I_{\text {ave }}=20 \mu \mathrm{A}$ of the component SQUID, the allowed spread in critical currents of Josephson junctions is $0.4 I_{\text {ave }}$ to $2.2 I_{\text {ave }}$ for achieving the low flux noise with the series SQUID array.
\end{abstract}

Index Terms-Critical-current spread, high-temperature superconductors, Josephson junctions, SQUID.

\section{INTRODUCTION}

$\mathbf{T}$ HE flux noise of the $N$-dc-SQUIDs array is expected to be less than a single dc SQUID by a factor of $N^{-0.5}$ [1], [2]. This characteristic is appealing for the magnetometer application. For the $N$ identical dc SQUID in series, the voltage modulation depth $V_{p p}$ should be amplified by a factor of $N$. And the white voltage noise of the array is $S_{v, \text { array }}=N S_{v}$, where $S_{v}$ is the voltage noise of each dc SQUID in the array. Hence, the resultant flux noise $S_{\Phi}^{1 / 2}$ of the SQUID array is [1], [2]

$$
S_{\Phi, \text { array }}^{1 / 2}=\left(\frac{\Phi_{0} S_{v}^{1 / 2}}{\frac{\partial V}{\partial \Phi}}\right) \cdot N^{-1 / 2}
$$

in which $\partial \mathrm{V} / \partial \Phi \approx \pi V_{p p} / \Phi_{0}$ is the flux-to-voltage transfer function of the component SQUID in the array. By using (1), the predicted field noise level of the SQUID-array magnetometer can be much less than that of the conventional dc SQUID. However, the experimental results [3], [4] did not show that the flux noise of the HTS series-dc-SQUID-array magnetometer could be less than that of the conventional HTS dc SQUID magnetometer. The possible reason for the discrepancy may be the spread of critical currents [4], [5] in the

Manuscript received October 3, 2004. This work was supported by the National Science Council of R.O.C. under Grant NSC91-2119-M-027-001, Grant NSC92-2112-M-027-004, and Grant NSC93-2112-M-027-003.

J. T. Jeng, H. C. Hung, and C. R. Lin are with the Institute of Mechatronic Engineering, National Taipei University of Technology, Taipei 106, Taiwan (e-mail: jtjeng@ntut.edu.tw).

C. H. Wu, K. L Chen, J.-C. Chen, and H. C. Yang are with the Department of Physics, National Taiwan University, Taipei 106, Taiwan (e-mail: hcyang@ phys.ntu.edu.tw).

S. H. Liao and H. E. Horng are with the Department of Physics, National Taiwan Normal University, Taipei 116, Taiwan (e-mail: phyfv001@ scc.ntnu.edu.tw).

Digital Object Identifier 10.1109/TASC.2005.850063 paralleled grain-boundary Josephson junctions of the HTS dc SQUID. The details about the effect of critical-current spread on $\partial \mathrm{V} / \partial \Phi$ of the HTS SQUID array are not yet clear. In this report, we investigate the effect of the critical-current spread of the grain-boundary Josephson junctions on the voltage swing of the HTS series-dc-SQUID array. The dispersion in critical currents as well as the asymmetry of the component SQUID is taken into account in calculating the voltage swing and the flux noise of the array. The allowed level of the spread in critical currents to achieve the low flux noise is predicted.

\section{Simulation For HTS SERIES dc SQUID ARRAY}

\section{A. Assumptions for the HTS Series dc SQUID Array}

In simulating the voltage-flux modulation curve of the HTS series dc SQUID array, we assumed that:

1. There is a small but nonnegligible spread in the effective area of the SQUID. The spread is a Gaussian distribution.

2. All of the SQUIDs are geometrically symmetric.

3. The critical current in the grain-boundary Josephson junctions has the logarithmic spread [5].

4. The critical current $I_{0}$ and the resistance $R_{0}$ of the grain-boundary Josephson junctions show the scaling behavior [6] $I_{0} R_{0} \propto\left(j_{\mathrm{c}}\right)^{0.5}$, in which $j_{\mathrm{c}}$ is the critical current density of the junction.

5. The voltage-current $(V-I)$ characteristic curve of the SQUID follows the Ambegaokar-Halperin's expression [7] while the critical current is modulated by the magnetic flux $\Phi$.

6. The intrinsic critical current asymmetry results in a flux shift [8] in the voltage-flux $(V-\Phi)$ curve by $\Delta \Phi=0.5\left(\alpha_{I} \beta_{L}\right) \Phi_{0}$, where $\alpha_{I}$ is the critical current asymmetry parameter, $\beta_{L}=2 L I_{0} / \Phi_{0}$ is the screening parameter, $\Phi_{0}$ is the flux quantum, and $L$ is the SQUID inductance.

7. The spread in the junction resistance is small, and the flux-shift due to the resistance asymmetry is negligible.

8. The interaction between the adjacent SQUIDs in the array owing to inductive coupling is negligible.

9. The white voltage noise of the dc SQUID follows that of the Josephson junctions in parallel [9].

10. The flux-to-voltage transfer function of the SQUID array is $\pi V_{p p, a r r a y} / \Phi_{0}$, where $V_{p p, a r r a y}$ is the maximum voltage swing of the SQUID array. 


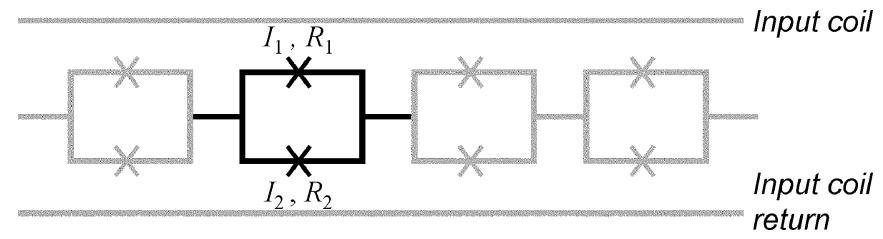

Fig. 1. Schematics of the HTS dc SQUID array with co-planar input coils.

Note that with the assumption 5 , it implies the capacitance of the Josephson junction is neglected, the current-phase relation is sinusoidal, and no excess supercurrent exists in the grain-boundary Josephson junctions.

The schematic diagram of the HTS de SQUID array made from the single-layered superconductor film is shown in Fig. 1. Generally, the critical currents $I_{1}$ and $I_{2}$ as well as the resistance $R_{1}$ and $R_{2}$ of the Josephson junctions in the component SQUID are intrinsically asymmetric. The degrees of asymmetry in the critical currents and the resistances are represented by the asymmetric parameters $\alpha_{I}$ and $\alpha_{R}$ respectively as follows:

$$
\begin{aligned}
I_{1} & =\left(1+\alpha_{I}\right) I_{0}, \quad I_{2}=\left(1-\alpha_{I}\right) I_{0} \\
R_{1} & =\frac{R_{0}}{\left(1-\alpha_{R}\right)}, \quad R_{2}=\frac{R_{0}}{\left(1+\alpha_{R}\right)},
\end{aligned}
$$

in which $I_{0}=\left(I_{1}+I_{1}\right) / 2$, and $R_{0}=2 R_{1} R_{2} /\left(R_{1}+R_{2}\right)$. The allowed values of $\alpha_{I}$ and $\alpha_{R}$ are from -1 to 1 . Since we have assumed the geometrical symmetry in assumption 2, the asymmetry in critical currents and resistance are respectively caused by the intrinsic asymmetry in the critical current density $j_{\mathrm{c}}$ and the contact resistance $\rho_{\text {contact }}$ of the grain-boundary Josephson junctions [8].

\section{B. Critical-Current-Modulation Model}

When the self-induced flux is negligible, i.e. the screening parameter $\beta_{L} \ll 1$, the critical current modulation of the asymmetric dc SQUID can be written as follows [10]:

$$
I_{c}(\Phi)=2 I_{0} \sqrt{1-\left(1-\alpha_{I}^{2}\right) \sin ^{2}\left(\frac{\pi \Phi}{\Phi_{0}}\right)}
$$

in which $I_{0}$ is the critical current of the Josephson junctions, $\alpha_{I}$ is the critical-current-asymmetry parameter, $\Phi_{0}$ is the magnetic flux quantum, and $\Phi$ is the applied magnetic flux. For $\alpha_{I}=0$, (3) reduces to the one for the symmetric dc SQUID $I_{\mathrm{c}}(\Phi)=2 I_{0}\left|\cos \left(\pi \Phi / \Phi_{0}\right)\right|$. When the SQUID inductance is large, (3) no longer holds as the self-induced flux is nonnegligible. The critical current modulation amplitude is further reduced from the prediction of (3) due to the self-screening effect. As the amount of the self-induced flux can not be solved analytically, there is no explicit expression for $I_{\mathrm{c}}(\Phi)$ with the nonnegligible SQUID inductance [10]. However, since (3) demonstrates the main feature of the critical-current modulation, it may be possible to adapt (3) for the approximation of $I_{\mathrm{c}}(\Phi)$ in the large inductance case. To do this, we added the dimensionless screening factor $f_{s}$ to the sinusoidal term in (3) as follows:

$$
I_{c}(\Phi)=2 I_{0} \sqrt{1-\left(1-\alpha_{I}^{2}\right) \sin ^{2}\left(\frac{\pi \Phi}{\Phi_{0}}\right) \cdot f_{s}}
$$

For the consistency with (3), the value of $f_{\mathrm{s}}$ must be $f_{\mathrm{s}}=1$ in the small inductance case. For the very large inductance case, the modulation amplitude of the critical current reduces to zero, which sets the limit $f_{\mathrm{s}} \geq 0$. Hence, the value of $f_{\mathrm{s}}$ should vary monotonically from 1 to 0 when the SQUID inductance increases from 0 to infinite. The screening factor $f_{\mathrm{s}}$ should be a function of the screening parameter $\beta_{L}$ for the inductance dependence. In addition, it could be a function of the noise parameter $\Gamma$ since the thermal noise may reduce the critical-current-modulation amplitude of the SQUID.

To find the appropriate expression for $f_{\mathrm{S}}\left(\beta_{L}, \Gamma\right)$, one may check the validity of the critical current modulation function in (4). To do this, we assumed the $V-I$ characteristic curve of the SQUID follows that of the over-damped Josephson junction with thermal noise. The equation found by Ambegaokar and Halperin [7] was used to calculate the $V-I$ curve of the SQUID:

$$
\begin{gathered}
V(I)=0.5 I_{c} R_{0} \pi \Gamma_{a}\left\{\left(e^{4 \pi x / \Gamma_{a}}-1\right)^{-1}\left[\int_{0}^{2 \pi} g(\theta) d \theta\right]\left[\int_{0}^{2 \pi} \frac{d \theta^{\prime}}{g\left(\theta^{\prime}\right)}\right]\right. \\
\left.+\left[\int_{0}^{2 \pi} d \theta \int_{0}^{\theta} \frac{g(\theta)}{g\left(\theta^{\prime}\right)} d \theta^{\prime}\right]\right\}^{-1}
\end{gathered}
$$

in which $x=I / I_{c}, I$ is the current, and $\Gamma_{a}=2 \pi k_{\mathrm{B}} T / I_{\mathrm{c}} \Phi_{0}$ is the apparent noise parameter, $k_{\mathrm{B}}$ is the Boltzmann constant, and $T$ is the temperature. In the integrands, the function $g(\theta)=$ $\exp \left[2(x \theta+\cos \theta) / \Gamma_{a}\right]$, and $\theta, \theta^{\prime}$ are the dummy variables for numerical integration. The critical current $I_{\mathrm{c}}$ in $(5)$ is modulated by the magnetic flux $\Phi$ as given in (4). By using the (4) and (5), the voltage modulation depth $V_{p p}$ of the SQUID biased at $I=2 I_{0}$ is given as follows:

$$
V_{p p}=V\left(2 I_{0} ; \Phi=0.5 \Phi_{0}\right)-V\left(2 I_{0} ; \Phi=0\right)
$$

To find the form of $f_{\mathrm{s}}$ in terms of $\beta_{L}$ and $\Gamma$, we check the consistency of the modulation depth predicted by (6) with the known empirical formula for the symmetric dc SQUID reported by Enpuku et al. [11]

$$
V_{p p}=\frac{4 I_{0} R_{0}}{\pi\left(1+\beta_{L}\right)} \exp \left(-2.75 \Gamma \beta_{L}\right)
$$

and the one by Koelle et al. [12]:

$$
V_{p p}=\frac{I_{0} R_{0}\left(7.3 \beta_{L}^{0.15}\right)}{\pi\left(1+\beta_{L}\right)\left[\left(80 \Gamma \beta_{L}\right)^{0.4}+0.35\left(4 \Gamma \beta_{L}\right)^{2.5}\right]}
$$

With the aid of (7) and (8), the possible $f_{\mathrm{s}}\left(\beta_{L}, \Gamma\right)$ is found to be

$$
f_{s}\left(\beta_{L}, \Gamma\right)=\left\{1+\beta_{L}\left[1+\left(\Gamma \beta_{L}\right)^{2}\right]\right\}^{-1.5}
$$

The voltage modulation depths of the symmetric SQUID calculated with (7), (8), and (6) are shown in Fig. 2. For large $\beta_{L}$, we found that all the results are similar. For small $\beta_{L}$, the predictions of the three different equations differ. Since the equation in (4) reduces to (3) for small $\beta_{L}$, our model should be valid in this range. Our model is also in agreement with the empirical formula for the $V_{\mathrm{pp}}$ of asymmetric dc SQUID reported by 

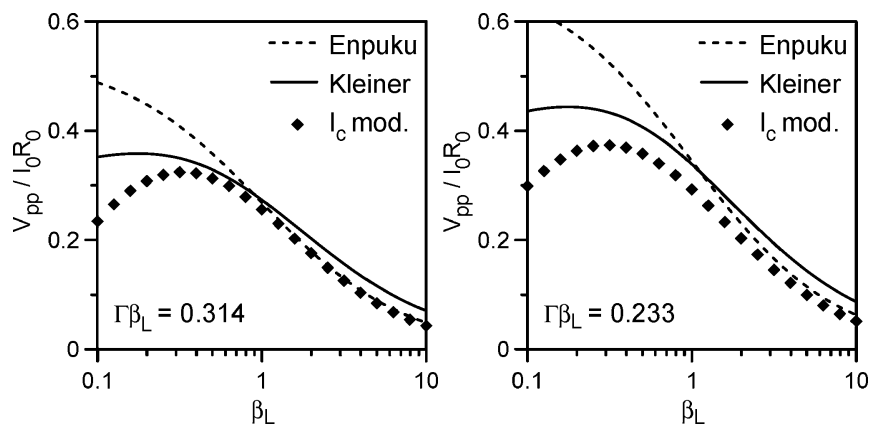

Fig. 2. Comparison in the predicted $V_{\text {pp }}$ of symmetric dc SQUID between the empirical formulas reported by Enkupu et al. (dashed curve), Koelle et al. (solid curve), and the critical current modulation model (solid diamonds).
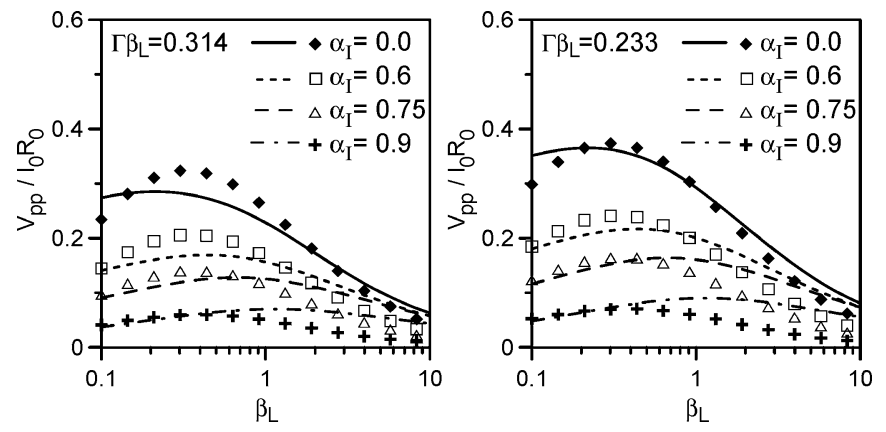

Fig. 3. Comparison in the predicted $V_{\mathrm{pp}}$ of asymmetric dc SQUID between the empirical formula reported by Müller et al. (curves) and the critical-currentmodulation model (symbols) for various $\alpha_{I}$.

Müller et al. [8]. The comparisons in the predicted $V_{\mathrm{pp}}$ 's between the their formula and our model are shown in Fig. 3.

\section{Simulation of Series dc SQUID Array}

The critical-current-modulation model described above was applied to simulate of $V-I$ and $V-\Phi$ curves the series-dcSQUID array with spread in critical currents of Josephson junctions. It is reported [5] that the spread in critical currents of the grain boundary Josephson junctions follows the log-normal distribution:

$$
P(\gamma)=\frac{1}{\sigma_{\ln I} \sqrt{2 \pi}} \exp \left[-0.5\left(\frac{\gamma}{\sigma_{\ln I}}\right)^{2}\right]
$$

in which $\sigma_{\ln I}$ is the logarithmic critical current spread [5] of the logarithmic critical current $\gamma=\ln \left(I_{0} / I_{\text {ave }}\right)$, and $I_{\text {ave }}=$ $\exp \left(\left\langle\ln I_{0}\right\rangle\right)$ is the logarithmic average of the critical current $I_{0}$ of all the Josephson junctions. In our simulation for the SQUID array the junction resistance $R_{0}$ is assumed to have the spread similar to (10), but the logarithmic average of the resistance $R_{\text {ave }}$ is determined by the scaling behavior $I_{\text {ave }} R_{\text {ave }} \propto\left(j_{\mathrm{c}}\right)^{0.5}$. The critical current of the $i$ 'th dc SQUID in terms of the logarithmic critical currents $\gamma_{1, i}$ and $\gamma_{2, i}$ of the Josephson junctions is

$$
2 I_{0, i}=I_{\text {ave }}\left[\exp \left(\gamma_{1, i}\right)+\exp \left(\gamma_{2, i}\right)\right]
$$

and the critical current asymmetry parameter $\alpha_{I, i}$ of the $i$ 'th SQUID becomes

$$
\alpha_{I, i}=\frac{\exp \left(\gamma_{1, i}\right)-\exp \left(\gamma_{2, i}\right)}{\exp \left(\gamma_{1, i}\right)+\exp \left(\gamma_{2, i}\right)}
$$
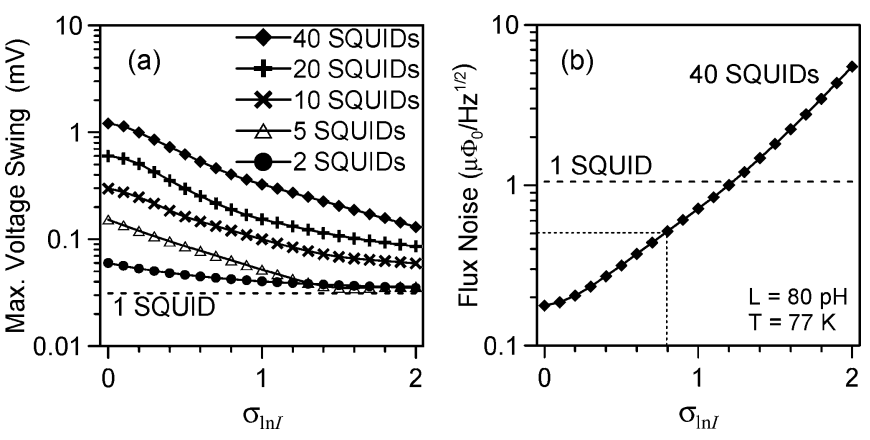

Fig. 4. Simulation results of, (a) the maximum voltage swing, (b) the flux noise, of the series SQUID array at $77 \mathrm{~K}$. The average critical current and resistance of the component SQUIDs are $2 I_{\text {ave }}=20 \mu \mathrm{A}$ and $0.5 R_{\text {ave }}=5 \Omega$. The resistance spread is assumed to be $\sigma_{\ln R}=0.1$.

The critical current asymmetry results in a flux shift in the $V-\Phi$ curve of the $i$ 'th SQUID by $\Delta \Phi=0.5\left(\alpha_{I, i} \beta_{L, i}\right) \Phi_{0}$, where $\beta_{L, i}$ is the screening parameter of the $i$ 'th SQUID. By taking all the effects described above into account, the $V-I$ and $V-\Phi$ curves of the series dc SQUID are obtained by summing up those of the component SQUIDs. Finally, the flux noise of the SQUID array is calculated with the following equation [9]:

$$
S_{\Phi, \text { array }}^{1 / 2}=\frac{\sqrt{\sum_{i=1}^{N}\left[1+0.5\left(\frac{I_{b}}{2 I_{0, i}}\right)^{2}\right]\left(\frac{4 k_{B} T R_{d, i}^{2}}{0.5 R_{0, i}}\right)}}{\left(\frac{\pi V_{p p, a r r a y}}{\Phi_{0}}\right)}
$$

in which $I_{\mathrm{b}}=2 I_{\text {ave }}$ is the bias current, and $V_{p p \text {,array }}$ is the maximum voltage swing of the SQUID array, $2 I_{0, i}$ is the critical current, $0.5 R_{0, i}$ is the normal state resistance, and $R_{d, i}$ is the dynamic resistance $(\mathrm{d} V / \mathrm{d} I)_{i}$ of the $i$ 'th SQUID.

The simulation results of the maximum voltage swing $V_{p p, a r r a y}$ and the flux noise of the SQUID arrays with $80-\mathrm{pH}$ inductance for each element are shown in Fig. 4 . The $V_{p p \text {,array }}$ decreases almost exponentially with the increased critical current spread $\sigma_{\ln I}$ as shown in Fig. 4(a). For the 40 SQUID array, the flux noise exhibits an exponential growth with the greater $\sigma_{\ln I}$ as shown in Fig. 4(b). Although the minimum flux noise of $0.17 \mu \Phi_{0} / \mathrm{Hz}^{1 / 2}$ is achieved with $\sigma_{\ln I}=0$, the flux noise grows with $\sigma_{\ln I}$ and reaches $1.0 \mu \Phi_{0} / \mathrm{Hz}^{1 / 2}$ at $\sigma_{\ln I}=1.2$, where it becomes the same as the single-SQUID curve. Since the voltage noise of the array was found to slightly increase by a factor of 1.3 from $\sigma_{\ln I}=0$ to $\sigma_{\ln I}=1.2$, the reduction in $V_{p p \text {,array }}$ should be responsible for the exponential growth in the flux noise.

As the critical-current spread is always nonzero, it is interesting whether the $\sigma_{\ln I}$ of the current junction technologies permit the flux-noise reduction with the HTS dc SQUID array. When the flux noise of the 40 SQUID array is half the noise of the single SQUID, the critical current spread is $\sigma_{\ln I}=0.8 \mathrm{ac}-$ cording to Fig. 4(b). This level corresponds to the on-chip spread in critical currents of $0.4 I_{\text {ave }}$ to $2.2 I_{\text {ave }}$.

\section{EXPERIMENTAL RESULTS}

The above simulation technique was applied to fit our experimental results of the 40 SQUID series array, which was made from the single-layered $\mathrm{YBa}_{2} \mathrm{Cu}_{3} \mathrm{O}_{\mathrm{y}}$ superconductor film on 

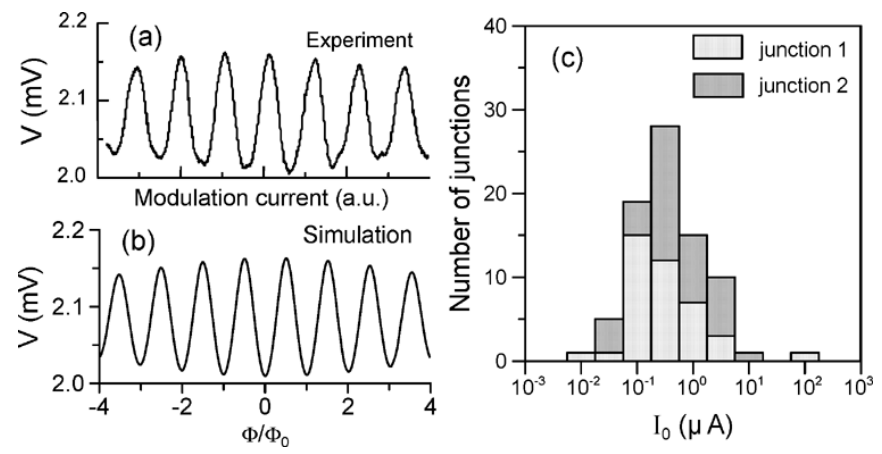

Fig. 5. (a) The observed voltage-flux curve of the 40 SQUID array fabricated on the bi-crystal substrate. The temperature was $55 \mathrm{~K}$, and the inductance of the component SQUID was estimated to be $80 \mathrm{pH}$. (b) The simulated voltage-flux curve with the critical current spread $\sigma_{\ln I}=1.43, I_{\text {ave }}=0.5 \mu \mathrm{A}$. (c) Distribution of the critical currents of the Josephson junctions.

the bi-crystal $\mathrm{SrTiO}_{3}$ substrate with $15^{\circ} / 15^{\circ}$ mis-orientation angle. The hole-size of the component SQUID is $29 \mu \mathrm{m} \times$ $30 \mu \mathrm{m}$. The line-widths are $8 \mu \mathrm{m}$ for the SQUID loop and $3 \mu \mathrm{m}$ for the junctions. The estimated SQUID inductance is $80 \mathrm{pH}$. Four dummy SQUID loops are put into the ends of the array to avoid the nonuniform modulation field in the active component SQUID. The $V-\Phi$ curve of the array measured by using the uniform modulation field generated with a 50-cm Helmholtz coil is shown in Fig. 5(a). The simulated voltage-flux curve of the array and the distribution of the simulated critical currents of the Josephson junctions are shown Fig. 5(b) and (c) respectively. Although the measured $V-I$ curve was almost linear with the total resistance of $2.4 \mathrm{k} \Omega$, the $V-\Phi$ curve with a maximum modulation depth of $150 \mu \mathrm{V}$ was observed with the bias current around $1 \mu \mathrm{A}$. The best-fit spread in critical current was found to be $\sigma_{\ln I}=1.43$ with $I_{\text {ave }}=0.5 \mu \mathrm{A}$. The range of critical current spread corresponds to the small $\beta_{L}$ from 0.009 to 0.16 . The small $\beta_{L}$ avoided the deterioration of the voltage modulation depth of array caused by the flux shift in the component SQUID. The reduction in the modulation amplitude at large modulation fields was found dominated by the spread in the SQUID's effective area, similar to the result for the LTS SQUID array reported in [13]. The best-fit spread in the effec- tive area to simulate the reduced modulation field at large modulation field is $3.3 \%$. According to the best-fit simulation result, the critical-current spread in our sample was too large to exhibit the low flux noise characteristic.

\section{CONCLUSION}

We proposed the critical-current-modulation model and found that it fits well with the known empirical formulas for the voltage swing of a HTS dc SQUID. With the critical-currentmodulation model, we found that the voltage swing of the array decreases about exponentially with the logarithmic critical current spread $\sigma_{\ln I}$, which results in the flux noise $S_{\Phi}^{1 / 2}$ being enhanced exponentially with $\sigma_{\ln I}$. For the 40 SQUID array at $77 \mathrm{~K}$ with $I_{\text {ave }}=20 \mu \mathrm{A}$, the allowed level of the on-chip critical-current spread in junctions is within $0.4 I_{\text {ave }}$ to $2.2 I_{\text {ave }}$ for achieving the low flux noise.

\section{REFERENCES}

[1] R. P. Welty and J. M. Martinis, IEEE Trans. Magn., vol. 27, p. 2924, 1991.

[2] K. G. Stawiasz and M. B. Ketchen, IEEE Trans. Appl. Supercond., vol. 3, p. 1808, 1993.

[3] S. G. Lee, Y. Huh, G. S. Park, I. S. Kim, Y. K. Park, and J. C. Park, IEEE Trans. Appl. Supercond., vol. 7, p. 3347, 1997.

[4] V. Schultze, V. Zakosarenko, R. Usselsteijn, J. Ramos, and H. G. Meyer, IEEE Trans. Appl. Supercond., vol. 9, p. 3279, 1999.

[5] P. Shadrin, C. L. Jia, and Y. Divin, IEEE Trans. Appl. Supercond., vol. 13 , p. $603,2003$.

[6] R. Gross, L. Alff, A. Beck, O. M. Froehlich, D. Koelle, and A. Marx, IEEE Trans. Appl. Supercond., vol. 7, p. 2929, 1997.

[7] V. Ambegaokar and B. I. Halperin, Phys. Rev. Lett., vol. 22, p. 1364, 1969.

[8] J. Müller, S. Weiss, R. Gross, R. Kleiner, and D. Koelle, IEEE Trans. Appl. Supercond., vol. 11, p. 912, 2001.

[9] J. Clarke, W. M. Goubau, and M. B. Ketchen, J. Low Temp. Phys., vol. 25, p. 99, 1976.

[10] T. Van Duzer and C. W. Turner, Principles of Superconductive Devices and Circuits. New York: Elsevier North Holland, 1981.

[11] K. Enpuku, G. Takita, T. Maruo, and T. Minotani, J. Appl. Phys., vol. 78, p. 3498, 1995.

[12] D. Koelle, R. Kleiner, F. Ludwig, E. Dantsker, and J. Clarke, Rev. Modern Phys., vol. 71, p. 631, 1999.

[13] K. Li and S. P. Hubbell, IEEE Trans. Appl. Supercond., vol. 5, p. 3255 , 1995. 